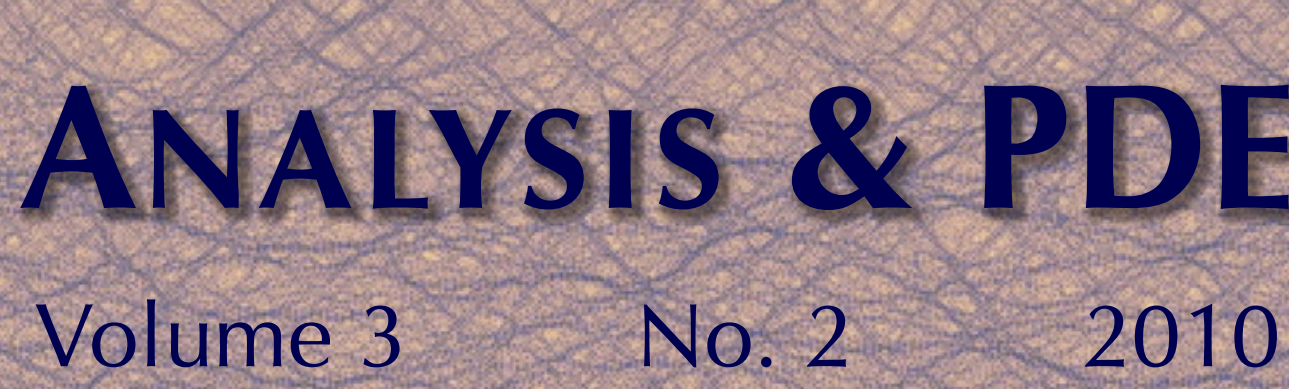

\author{
DONG LI AND XIAOYI ZHANG
}

REGULARITY OF ALMOST PERIODIC MODULO SCALING SOLUTIONS

FOR MASS-CRITICAL NLS AND APPLICATIONS 


\title{
REGULARITY OF ALMOST PERIODIC MODULO SCALING SOLUTIONS FOR MASS-CRITICAL NLS AND APPLICATIONS
}

\author{
DONG LI AND XIAOYI ZHANG
}

\begin{abstract}
We consider the $L_{x}^{2}$ solution $u$ to mass-critical NLS $i u_{t}+\Delta u= \pm|u|^{4 / d} u$. We prove that in dimensions $d \geq 4$, if the solution is spherically symmetric and is almost periodic modulo scaling, then it must lie in $H_{x}^{1+\varepsilon}$ for some $\varepsilon>0$. Moreover, the kinetic energy of the solution is localized uniformly in time. One important application of the theorem is a simplified proof of the scattering conjecture for mass-critical NLS without reducing to three enemies. As another important application, we establish a Liouville type result for $L_{x}^{2}$ initial data with ground state mass. We prove that if a radial $L_{x}^{2}$ solution to focusing masscritical problem has the ground state mass and does not scatter in both time directions, then it must be global and coincide with the solitary wave up to symmetries. Here the ground state is the unique, positive, radial solution to elliptic equation $\Delta Q-Q+Q^{1+4 / d}=0$. This is the first rigidity type result in scale invariant space $L_{x}^{2}$.
\end{abstract}

\section{Introduction}

Main results. We consider the $d$-dimensional mass-critical nonlinear Schrödinger equation

$$
i u_{t}+\Delta u=\mu|u|^{4 / d} u=: F(u) .
$$

Here, $\mu= \pm 1$, with $\mu=+1$ known as the defocusing and $\mu=-1$ as the focusing case. The name "mass-critical" refers to the fact that the scaling symmetry

$$
u(t, x)=\lambda^{d / 2} u\left(\lambda^{2} t, \lambda x\right)
$$

leaves both the equation and the mass invariant. Here the mass is defined as

$$
M(u(t))=\int_{\mathbb{R}^{d}}|u(t, x)|^{2} d x=M\left(u_{0}\right) .
$$

The precise meaning of the solution we discuss throughout the paper is the following:

Definition 1.1 (solution). A function $u: I \times \mathbb{R}^{d} \rightarrow \mathbb{C}$ on a nonempty time interval $I \subset \mathbb{R}$ is a strong $L_{x}^{2}\left(\mathbb{R}^{d}\right)$ solution (or solution for short) if it lies in the class $C_{t}^{0} L_{x}^{2}\left(K \times \mathbb{R}^{d}\right) \cap L_{t, x}^{2(d+2) / d}\left(K \times \mathbb{R}^{d}\right)$ for all compact $K \subset I$, and we have the Duhamel formula

$$
u\left(t_{1}\right)=e^{i\left(t_{1}-t_{0}\right) \Delta} u\left(t_{0}\right)-i \int_{t_{0}}^{t_{1}} e^{i\left(t_{1}-t\right) \Delta} F(u(t)) d t
$$

MSC2000: 35Q55.

Keywords: Schrödinger equation, mass-critical. 
for all $t_{0}, t_{1} \in I$. Here $e^{i t \Delta}$ is the propagator for free Schrödinger equation. We say that $u$ is a maximallifespan solution if the solution can not be extended to any strictly larger interval. We say that $u$ is global if $I=\mathbb{R}$.

The standard local theory for such solutions was worked out by Cazenave and Weissler [2003]. They constructed the local in time solution for arbitrary initial data in $L_{x}^{2}\left(\mathbb{R}^{d}\right)$. They also showed that the solution depends continuously on the initial data in the same space. However due to the criticality of the problem, the lifespan of the local solution depends on the profile of the initial data instead of the mere $L_{x}^{2}$-norm. When the initial data is small enough, they proved the solution exists globally and scatters in the following sense: there exist unique $u_{ \pm} \in L_{x}^{2}\left(\mathbb{R}^{d}\right)$ such that

$$
\lim _{t \rightarrow \infty}\left\|u(t)-e^{i t \Delta} u_{+}\right\|_{L_{x}^{2}}=\lim _{t \rightarrow-\infty}\left\|u(t)-e^{i t \Delta} u_{-}\right\|_{L_{x}^{2}}=0 .
$$

Whilst the local theory is fairly complete, the understanding of the global theory for large solutions is still only partial. Briefly speaking, the global theory for large solutions amounts to proving the global wellposedness and scattering for generic $L_{x}^{2}$ initial data in the defocusing case; investigating the long time behavior of global solutions, characterizing the structure and profile of finite time blowup solutions in the focusing case and so on. In recent years, by using concentration compactness tools developed and used in [Merle 1993; Kenig and Merle 2006; Keraani 2001; 2006; Bégout and Vargas 2007; Killip et al. 2008; 2009a; 2009b; Li and Zhang 2009b; 2009a], one can address part of these problems by exploring the properties of a large class of solutions which have certain compactness properties. To this end, following [Tao et al. 2008], we introduce:

Definition 1.2 (almost periodic modulo symmetry solutions). Let $u$ be the maximal-lifespan solution of (1-1) on time interval $I$. Let $I_{0} \subset I$ be a subinterval. We say $u$ is almost periodic modulo symmetries on $I_{0}$ if there exists functions $x(t), N(t), \xi(t), \theta(t)$ with $t \in I_{0}$ such that the orbit

$$
\left\{e^{i \theta(t)} e^{i x \cdot \xi(t)} N(t)^{-d / 2} u\left(t, \frac{x-x(t)}{N(t)}\right), t \in I_{0}\right\}
$$

is precompact in $L_{x}^{2}\left(\mathbb{R}^{d}\right)$. By the Arzelà-Ascoli Theorem, an equivalent way to write this definition is the following: there exists a function $C(\eta)$ such that for any $\eta>0$,

$$
\int_{|x-x(t)|>C(\eta) / N(t)}|u(t, x)|^{2} d x \leq \eta, \quad \int_{|\xi-\xi(t)|>C(\eta) N(t)}|\hat{u}(t, \xi)|^{2} d \xi \leq \eta .
$$

In particular, we call $u$ is almost periodic modulo scaling on $I_{0}$ if, in this situation, $x(t)=\xi(t) \equiv 0$ for all $t \in I_{0}$.

The parameter $N(t)$ is the frequency scale. In the physical space, its reciprocal corresponds to the concentration size of the solution. The parameter $x(t), \xi(t)$ correspond to the center of mass at physical and frequency spaces respectively. Basically we have no a priori control on these parameters, which is the main source of the difficulty of establishing useful properties for almost periodic modulo symmetry solutions. However, under the spherical symmetry assumption, one is allowed to fix the center of mass, thus leaving only one parameter $N(t)$ which can still vary arbitrarily. This case turns out to be treatable in high dimensions $d \geq 4$. Here is the main theorem of this paper: 
Theorem 1.3. Let $d \geq 4$. Let $u$ be a maximal-lifespan solution on $I$ and is spherically symmetric. Suppose $u$ is almost periodic modulo scaling on $I$. Then there exists $\varepsilon=\varepsilon(d)<4 / d$ such that

$$
u(t) \in H_{x}^{1+\varepsilon} \quad \text { for all } t \in I \text {. }
$$

Moreover, the kinetic energy of the solution is localized uniformly in time: for any $\eta>0$, there exists $C(\eta)$ such that for any $t \in I$

$$
\int_{|x| \geq C(\eta)}|\nabla u(t, x)|^{2} d x \leq \eta .
$$

Here, $\varepsilon$ only depends on the dimension $d$, while $C(\eta)$ depends also on the solution $u$.

Remark 1.4. This result seems a bit surprising in view of the fact that the scaling parameter $N(t)$ can vary arbitrarily and the solution is only assumed to be in the scale invariant space $L_{x}^{2}$. On the other hand, Theorem 1.3 bears similarities with previous works [Killip et al. 2008; 2009a; 2009b; Li and Zhang 2009b], where they were able to deal with dimensions two and higher. However in [Killip et al. 2009a; $\mathrm{Li}$ and Zhang 2009b], the solution is assumed to have $H_{x}^{1}$ regularity and this latter fact allows one to treat solutions being almost periodic modulo scaling in only one time direction. In [Killip et al. 2008; 2009b], the additional regularity is only established for three typical solutions known as three enemies. Namely, these are almost periodic modulo scaling solutions with a priori control on $N(t)$ :

(a) The self-similar solution. This solution is defined on maximal time interval $(0, \infty)$ and $N(t)=t^{-1 / 2}$ for any $t \in(0, \infty)$.

(b) The soliton-like solution. This solution is global and $N(t)=1$.

(c) The high to low cascade. This solution is also global with $N(t)$ satisfying the conditions $N(t) \leq 1$ and $\liminf _{t \rightarrow \pm \infty} N(t)=0$.

On the other hand, the technique in this paper allows us to deal with all enemies with no a priori assumption on $N(t)$ in dimensions $d \geq 4$.

Remark 1.5. The dependence on the dimension comes from the fact that in dimension $d \geq 4$, the nonlinearity $|u|^{4 / d} u$ can be put in Lebesgue space $L_{x}^{p}\left(\mathbb{R}^{d}\right)$ for some $p \geq 1$ only knowing that $u \in L_{x}^{2}\left(\mathbb{R}^{d}\right)$. This property is not available in low dimensions $d=2,3$. So in these dimensions, it is still open proving the additional regularity for solutions other than the three enemies.

Remark 1.6. Besides the spherical symmetry, we can also consider other symmetries that can freeze the center of mass at the origin. For example, one can consider the splitting spherical symmetry introduced in [ $\mathrm{Li}$ and Zhang 2009b]. In [ $\mathrm{Li}$ and Zhang 2009a], we select the six dimensions as a sample case to show how the technique can be extended to deal with the solution with splitting spherical symmetry and is almost periodic modulo scaling. There the main difficulty comes from the fact that the waves can propagate anisotropically along splitting subspaces. As shown in the proofs of Proposition 4.4 and Proposition 4.6, the spherical symmetry is mainly used to treat the part where the plane waves travel away from the origin. For this part, one uses the weighted Strichartz estimate for radial functions to get the decay. In the splittingly spherical symmetric case, we develop tools such as weighted Strichartz estimate (see [Li and Zhang 2009b]) for splittingly spherical symmetric functions to make use of the decay property. 
Remark 1.7. To prove Theorem 1.3 we need to control the parts of the solution both near the spatial origin and away from it. To control the part away from the origin, we use the techniques from [Killip et al. 2009a] where we need the radial assumption on the solution. To control the part near the origin, we introduce a novel local iteration scheme which actually does not need the radial assumption provided we already have the control on the piece away from the origin. We should also stress that our proof uses the almost periodicity in a very light way. Instead of assuming the solution is almost periodic modulo scaling on the whole time interval, one could assume the following sequential almost periodicity: there exist $t_{n}^{+} \rightarrow \sup I, t_{n}^{-} \rightarrow \inf I$ and scaling parameters $N\left(t_{n}^{+}\right), N\left(t_{n}^{-}\right)$, such that both of the sets

$$
\left\{N\left(t_{n}^{+}\right)^{-d / 2} u\left(t_{n}^{+}, \cdot / N\left(t_{n}^{+}\right)\right)\right\},\left\{N\left(t_{n}^{-}\right)^{-d / 2} u\left(t_{n}^{-}, \cdot / N\left(t_{n}^{-}\right)\right)\right\}
$$

are precompact in $L_{x}^{2}\left(\mathbb{R}^{d}\right)$.

Applications of Theorem 1.3. The applications of Theorem 1.3 are related to the scattering conjecture and the rigidity conjecture which we now explain. In the defocusing case, the scattering conjecture says that all solutions with finite mass exist globally and scatter in both time directions. In the focusing case, besides scattering solutions, there exist finite time blowup solutions as shown in [Glassey 1977] and the solitary wave solutions of the form $e^{i t} R(x)$. Here $R$ solves the elliptic equation

$$
\Delta R-R+|R|^{4 / d} R=0 .
$$

There are infinitely many solutions to this equation, but only one positive solution which is spherically symmetric (up to translations) and whose mass is minimal among all these $R^{\prime} s$. This solution is usually called the ground state:

Definition 1.8 (ground state [Berestycki and Lions 1979; Kwong 1989]). The ground state $Q$ refers to the unique positive radial Schwartz solution to the elliptic equation

$$
\Delta Q-Q+|Q|^{4 / d} Q=0
$$

It is believed that the mass of $Q$ serves as the minimal mass among all the nonscattering solutions in the focusing case. To summarize, we have:

Conjecture 1.9 (scattering conjecture). Let $u_{0} \in L_{x}^{2}\left(\mathbb{R}^{d}\right)$. In the focusing case, we also assume $M\left(u_{0}\right)<$ $M(Q)$. Then the corresponding solution to (1-1) exists globally and scatters in both time directions.

This conjecture has been proved in dimensions $d \geq 2$ when the initial data is spherically symmetric; see [Killip et al. 2008; 2009b]. ${ }^{1}$ We now give a high level overview of the proof which is based on a contradiction argument. Assuming the scattering conjecture is not true, one can then use concentration compactness tools to obtain minimal mass nonscattering ${ }^{2}$ solutions which are almost periodic modulo scaling (due to the spherical symmetry) with scaling parameter $N(t)$. To obtain better control of $N(t)$, another limiting procedure is performed to reduce the consideration to three typical solutions alluded as to "three enemies". To kill three enemies and thereby obtaining the contradiction, one can use the

\footnotetext{
${ }^{1}$ In the defocusing case and $d \geq 3$, one can take advantage of Morawetz estimate to prove the additional regularity; see [Tao et al. 2007] for more details.

${ }^{2}$ Here by "nonscattering", we mean that the $L_{t, x}^{2(d+2) / d}$ norm of the solution is infinite. Obviously, a "nonscattering" solution may blow up at finite time or exist globally with infinite $L_{t, x}^{2(d+2) / d}$ norm.
} 
information of $N(t)$ to obtain additional regularity of these solutions which together with a truncated virial argument establishes the claim.

Thanks to Theorem 1.3, we can simplify the argument by directly working with all enemies whose scaling parameter $N(t)$ can vary arbitrarily in dimensions $d \geq 4$. In other words, the limiting procedure of picking three enemies is not needed here. We record the result as:

Corollary 1.10 (scattering in dimension $d \geq 4$ with spherical symmetry). Let $d \geq 4$. Let $u_{0} \in L_{x}^{2}\left(\mathbb{R}^{d}\right)$ be spherically symmetric. In the focusing case, we assume $M\left(u_{0}\right)<M(Q)$. Then the solution to (1-1) with this initial data exists globally and satisfies

$$
\|u\|_{L_{t, x}^{2(d+2) / d}\left(\mathbb{R} \times \mathbb{R}^{d}\right)} \leq C\left(\left\|u_{0}\right\|_{L_{x}^{2}}\right) .
$$

We turn now to the rigidity conjecture.

In the focusing case, a main issue is to understand the large time behavior of nonscattering solutions. This problem has only been addressed in the case when the mass of $u$ is equal to or slightly bigger than that of the ground state; see [Merle 1993; Merle and Raphael 2005; Killip et al. 2009a; Li and Zhang 2009b] and the references therein. In this paper, we are primarily concerned with the case when the solution has the ground state mass. Our main focus is to characterize and classify all such solutions. At the level of ground state mass, there are two explicit examples of nonscattering solutions: the solitary wave SW which exists globally and the pseudoconformal ground state $\operatorname{Pc}(Q)$ which blows up at $t=0$ :

$$
\mathrm{SW}=e^{i t} Q(x), \quad \operatorname{Pc}(Q)=|t|^{-d / 2} e^{\left(i|x|^{2}-4\right) /(4 t)} Q\left(\frac{x}{t}\right) .
$$

It is conjectured that, up to symmetries, these are the only two threshold solutions for scattering at the level of minimal mass. Associated with this is the following rigidity conjecture which identifies all solutions with ground state mass as either $\mathrm{SW}$ or $\operatorname{Pc}(Q)$ if they do not scatter. Since both mass and the equation are invariant under a couple of symmetries, the coincidence of the solutions with the examples only hold modulo these symmetries. Specifically, the symmetries are: translation, phase rotation, scaling and the Galilean boost.

Conjecture 1.11 (rigidity conjecture at the ground state mass). Let $u_{0} \in L_{x}^{2}\left(\mathbb{R}^{d}\right)$ satisfy $M\left(u_{0}\right)=M(Q)$. Then only the following cases can occur:

(1) The solution u blows up at finite time, then in this case u must coincide with $\operatorname{Pc}(Q)$ up to symmetries of the equation.

(2) The solution $u$ is a global solution. Then in this case, $u$ either scatters in both time directions or $u$ must coincide with $\mathrm{SW}$ up to symmetries of the equation.

Merle [1993] considered the first part of the conjecture, where he identified all finite time blowup solutions as $\operatorname{Pc}(Q)$ under an additional $H_{x}^{1}$ assumption on the initial data. See also [Weinstein 1986] for the preliminary result and [Hmidi and Keraani 2005] for a simplified proof of Merle's argument. By Merle's result and pseudoconformal transformation, the second part of the conjecture, which characterizes all global solutions with ground state mass, still holds if we make the strong assumption that the initial data $u_{0} \in \Sigma=\left\{f \in H_{x}^{1}, x f \in L_{x}^{2}\right\}$. Finally it is worthwhile noticing that Merle's argument works for all dimensions without any symmetry assumption on the initial data. 
Without the $\Sigma$ assumption on the initial data, it is not clear at all how to deal with the case when $u_{0}$ is merely in $L_{x}^{2}$ and the corresponding solution is global. Recently in [Killip et al. 2009a; Li and Zhang $2009 \mathrm{~b}]$, we proved the second part of the conjecture when the initial data $u_{0} \in H_{x}^{1}\left(\mathbb{R}^{d}\right), d \geq 2$ and is spherically symmetric. In dimensions $d \geq 4$, the results hold even under a weaker symmetry assumption, namely, the initial data is only required to be splitting-spherical symmetric (see [Li and Zhang 2009b] for more details).

As stated, all the results concerning the rigidity conjecture require the $H_{x}^{1}$ regularity on the initial data since it is the minimal regularity to define the energy and to carry out the spectral analysis. Here the energy refers to

$$
E(u(t))=\frac{1}{2}\|\nabla u(t)\|_{L_{x}^{2}}^{2}-\frac{d}{2(d+2)}\|u(t)\|_{L_{x}^{2(d+2) / d}}^{2(d+2) / d}=E\left(u_{0}\right) .
$$

To prove the rigidity results for pure $L_{x}^{2}$ solutions, a reasonable strategy is to upgrade the regularity of the solution to $H_{x}^{1}$ or better by taking advantage of certain compactness properties of the solutions. This is where Theorem 1.3 has to be used. We can then use known $H_{x}^{1}$ results to classify these solutions. Therefore as a direct consequence of Theorem 1.3, we have:

Theorem 1.12 (rigidity for two-way nonscattering solutions with ground state mass). Let $d \geq 4$. Let $u_{0} \in L_{x}^{2}\left(\mathbb{R}^{d}\right)$ be spherically symmetric and $M\left(u_{0}\right)=M(Q)$. Let $u$ be the maximal lifespan solution on $I$ which does not scatter on both sides:

$$
\|u\|_{L_{t, x}^{2(d+2) / d}\left(\left[t_{0}, \sup I\right) \times \mathbb{R}^{d}\right)}=\|u\|_{L_{t, x}^{2(d+2) / d}\left(\left(\inf I, t_{0}\right] \times \mathbb{R}^{d}\right)}=\infty, t_{0} \in I .
$$

Then $I=\mathbb{R}$ and $u=e^{i t} Q$ up to phase rotation and scaling.

For technical reasons, we need to impose the condition that the solution does not scatter in both time directions. It is an interesting problem to extend our techniques to the case when the solution scatters only in one time direction, but does not scatter in the other.

We give the proof of these two results in Section 3. Now we briefly sketch the proof of Theorem 1.3.

Main idea of the proof of Theorem 1.3: a local iteration scheme. We will work with each single dyadic frequency of $u$ :

$$
\left\|P_{N} u(t)\right\|_{L_{x}^{2}} \text {. }
$$

The decay in $N$ will correspond to the regularity of the solution. First we observe that when restricted to the region away from the origin, the argument in [Killip et al. 2009a] gives us

$$
\left\|\phi_{>1} P_{N} u(t)\right\|_{L_{x}^{2}} \lesssim N^{-1-\varepsilon}
$$

with a uniform in time bound. Here $\phi_{>1}$ is a smooth cut-off function supported in the region $|x|>1$. This reduces matters to estimating the part of the solution near the spatial origin, that is, $\left\|\phi_{\leq 1} P_{N} u(t)\right\|_{L_{x}^{2}}$. This piece is trivially bounded by

$$
A_{N}=\left\|P_{N} u\right\|_{S([t, t+1 / \sqrt{N}])},
$$

that is, the Strichartz norm of $P_{N} u$ on a local time interval $[t, t+1 / \sqrt{N}]$. It turns out, after some technical manipulations, that this latter quantity is better suited for iteration and bootstrapping. Indeed 
we shall establish recurrent relations for $A_{N}$ and we will iterate our estimates only finitely many (but sufficiently many) steps. The crucial point is that during the iteration process, we shall never need more than the information of the solution on a unit time interval $[t, t+1]$. Therefore we do not need to use the full control of $N(t)$. We remark that although as a sacrifice the $H_{x}^{1+\varepsilon}$ norm of $u(t)$ depends on $t$, this information combined with the kinetic energy localization in Section 3 suffice to prove Corollary 1.10 and Theorem 1.12.

\section{Preliminaries}

Some notation. We write $X \lesssim Y$ or $Y \gtrsim X$ to indicate $X \leq C Y$ for some constant $C>0$. We use $O(Y)$ to denote any quantity $X$ such that $|X| \lesssim Y$. We use the notation $X \sim Y$ whenever $X \lesssim Y \lesssim X$. The fact that these constants depend upon the dimension $d$ will be suppressed. If $C$ depends upon some additional parameters, we will indicate this with subscripts; for example, $X \lesssim_{u} Y$ denotes the assertion that $X \leq C_{u} Y$ for some $C_{u}$ depending on $u$. Sometimes when the context is clear, we will suppress the dependence on $u$ and write $X \lesssim_{u} Y$ as $X \lesssim Y$. We will write $C=C\left(Y_{1}, \ldots, Y_{n}\right)$ to stress that the constant $C$ depends on quantities $Y_{1}, \ldots, Y_{n}$. We denote by $X \pm$ any quantity of the form $X \pm \varepsilon$ for any $\varepsilon>0$.

We use the "Japanese bracket" convention: $\langle x\rangle:=\left(1+|x|^{2}\right)^{1 / 2}$.

We write $L_{t}^{q} L_{x}^{r}$ to denote the Banach space with norm

$$
\|u\|_{L_{t}^{q} L_{x}^{r}\left(\mathbb{R} \times \mathbb{R}^{d}\right)}:=\left(\int_{\mathbb{R}}\left(\int_{\mathbb{R}^{d}}|u(t, x)|^{r} d x\right)^{q / r} d t\right)^{1 / q},
$$

with the usual modifications when $q$ or $r$ are equal to infinity, or when the domain $\mathbb{R} \times \mathbb{R}^{d}$ is replaced by a smaller region of spacetime such as $I \times \mathbb{R}^{d}$. When $q=r$ we abbreviate $L_{t}^{q} L_{x}^{q}$ as $L_{t, x}^{q}$.

Throughout this paper, we will use $\phi \in C^{\infty}\left(\mathbb{R}^{d}\right)$ for a radial bump function supported in the ball $\left\{x \in \mathbb{R}^{d}:|x| \leq 25 / 24\right\}$ and equal to 1 on the ball $\left\{x \in \mathbb{R}^{d}:|x| \leq 1\right\}$. For any constant $C>0$, we set $\phi_{\leq C}(x):=\phi(x / C)$ and $\phi_{>C}:=1-\phi_{\leq C}$.

Basic harmonic analysis. For each number $N>0$, we define the Fourier multipliers

$$
\begin{aligned}
\widehat{P_{\leq N} f}(\xi) & :=\phi_{\leq N}(\xi) \hat{f}(\xi), \\
\widehat{P_{>N} f}(\xi) & :=\phi_{>N}(\xi) \hat{f}(\xi), \\
\widehat{P_{N} f}(\xi) & :=\left(\phi_{\leq N}-\phi_{\leq N / 2}\right)(\xi) \hat{f}(\xi),
\end{aligned}
$$

and similarly $P_{<N}$ and $P_{\geq N}$. We also define

$$
P_{M<\cdots \leq N}:=P_{\leq N}-P_{\leq M}=\sum_{M<N^{\prime} \leq N} P_{N^{\prime}}
$$

whenever $M<N$. We will usually use these multipliers when $M$ and $N$ are dyadic numbers (that is, of the form $2^{n}$ for some integer $n$ ); in particular, all summations over $N$ or $M$ are understood to be over dyadic numbers. Nevertheless, it will occasionally be convenient to allow $M$ and $N$ not to be powers of 2 . Since $P_{N}$ is not truly a projection $\left(P_{N}^{2} \neq P_{N}\right)$, we will occasionally need to use fattened Littlewood-Paley 
operators:

$$
\tilde{P}_{N}:=P_{N / 2}+P_{N}+P_{2 N}
$$

These obey $P_{N} \tilde{P}_{N}=\tilde{P}_{N} P_{N}=P_{N}$.

Like all Fourier multipliers, the Littlewood-Paley operators commute with the propagator $e^{i t \Delta}$, as well as with differential operators such as $i \partial_{t}+\Delta$. We will use basic properties of these operators many times, including:

Lemma 2.1 (Bernstein estimates). For $1 \leq p \leq q \leq \infty$,

$$
\begin{aligned}
\left\||\nabla|^{ \pm s} P_{N} f\right\|_{L_{x}^{p}\left(\mathbb{R}^{d}\right)} & \sim N^{ \pm s}\left\|P_{N} f\right\|_{L_{x}^{p}\left(\mathbb{R}^{d}\right)}, \\
\left\|P_{\leq N} f\right\|_{L_{x}^{q}\left(\mathbb{R}^{d}\right)} & \lesssim N^{d / p-d / q}\left\|P_{\leq N} f\right\|_{L_{x}^{p}\left(\mathbb{R}^{d}\right)}, \\
\left\|P_{N} f\right\|_{L_{x}^{q}\left(\mathbb{R}^{d}\right)} & \lesssim N^{d / p-d / q}\left\|P_{N} f\right\|_{L_{x}^{p}\left(\mathbb{R}^{d}\right)} .
\end{aligned}
$$

While it is true that spatial cutoffs do not commute with Littlewood-Paley operators, we still have the following:

Lemma 2.2 (mismatch estimates in real space). Let $R, N>0$. Then

$$
\begin{gathered}
\left\|\phi_{>R} \nabla P_{\leq N} \phi_{\leq R / 2} f\right\|_{L_{x}^{p}\left(\mathbb{R}^{d}\right)} \\
\left\|m N^{1-m} R^{-m}\right\| f \|_{L_{x}^{p}\left(\mathbb{R}^{d}\right)}, \\
\left\|\phi_{>R} P_{\leq N} \phi_{\leq R / 2} f\right\|_{L_{x}^{p}\left(\mathbb{R}^{d}\right)} \lesssim m N^{-m} R^{-m}\|f\|_{L_{x}^{p}\left(\mathbb{R}^{d}\right)}
\end{gathered}
$$

for any $1 \leq p \leq \infty$ and $m \geq 0$.

Proof. We will only prove the first inequality; the second follows similarly.

It is not hard to obtain kernel estimates for the operator $\phi_{>R} \nabla P_{\leq N} \phi_{\leq R / 2}$. Indeed, an exercise in nonstationary phase shows

$$
\left|\phi_{>R} \nabla P_{\leq N} \phi_{\leq R / 2}(x, y)\right| \lesssim N^{d+1-2 k}|x-y|^{-2 k} \phi_{|x-y|>R / 2}
$$

for any $k \geq 0$. An application of Young's inequality yields the claim.

Similar estimates hold when the roles of the frequency and physical spaces are interchanged. The proof is easiest when working on $L_{x}^{2}$, which is the case we will need; nevertheless, the following statement holds on $L_{x}^{p}$ for any $1 \leq p \leq \infty$.

Lemma 2.3 (mismatch estimates in frequency space). For $R>0$ and $N, M>0$ such that $\max \{N, M\} \geq$ $4 \min \{N, M\}$,

$$
\begin{aligned}
\left\|P_{N} \phi_{\leq R} P_{M} f\right\|_{L_{x}^{2}\left(\mathbb{R}^{d}\right)} & { }_{m} \max \{N, M\}^{-m} R^{-m}\|f\|_{L_{x}^{2}\left(\mathbb{R}^{d}\right)}, \\
\left\|P_{N} \phi_{\leq R} \nabla P_{M} f\right\|_{L_{x}^{2}\left(\mathbb{R}^{d}\right)} & { }_{m} M \max \{N, M\}^{-m} R^{-m}\|f\|_{L_{x}^{2}\left(\mathbb{R}^{d}\right)}
\end{aligned}
$$

for any $m \geq 0$. The same estimates hold if we replace $\phi_{\leq R}$ by $\phi_{>R}$.

Proof. The first claim follows from Plancherel's Theorem and Lemma 2.2 and its adjoint. To obtain the second claim from this, we write

$$
P_{N} \phi_{\leq R} \nabla P_{M}=P_{N} \phi_{\leq R} P_{M} \nabla \tilde{P}_{M}
$$

and note that $\left\|\nabla \tilde{P}_{M}\right\|_{L_{x}^{2} \rightarrow L_{x}^{2}} \lesssim M$. 
Some analysis tools. We will need the following radial Sobolev embedding to exploit the decay property of a radial function. For the proof and the more complete version, see [Tao et al. 2007].

Lemma 2.4 (radial Sobolev embedding [Tao et al. 2007]). Let the dimension d be at least 2. Let $s>0$, $\alpha>0$ and $1<p, q<\infty$ obey the scaling restriction $\alpha+s=d(1 / q-1 / p)$. Then the following holds:

$$
\left\||x|^{\alpha} f\right\|_{L^{p}\left(\mathbb{R}^{d}\right)} \lesssim\left\||\nabla|^{s} f\right\|_{L^{q}\left(\mathbb{R}^{d}\right)},
$$

where the implicit constant depends on $s, \alpha, p, q$. When $p=\infty$, we have

$$
\left\||x|^{(d-1) / 2} P_{N} f\right\|_{L^{\infty}\left(\mathbb{R}^{d}\right)} \lesssim N^{1 / 2}\left\|P_{N} f\right\|_{L_{x}^{2}\left(\mathbb{R}^{d}\right)} .
$$

We will need the following fractional chain rule lemma.

Lemma 2.5 (fractional chain rule for a $C^{1}$ function [Christ and Weinstein 1991; Staffilani 1997; Taylor 2000]). Let $G \in C^{1}(\mathbb{C}), \sigma \in(0,1)$, and $1<r, r_{1}, r_{2}<\infty$ such that $1 / r=1 / r_{1}+1 / r_{2}$. Then we have

$$
\left\||\nabla|{ }^{\sigma} G(u)\right\|_{r} \lesssim\left\|G^{\prime}(u)\right\|_{r_{1}}\left\||\nabla|^{\sigma} u\right\|_{r_{2}} .
$$

Proof. See [Christ and Weinstein 1991; Staffilani 1997; Taylor 2000].

Lemma 2.6 [Killip et al. 2008]. Let $0<s<1+4 / d$ and $F(u)=|u|^{4 / d} u$. Then

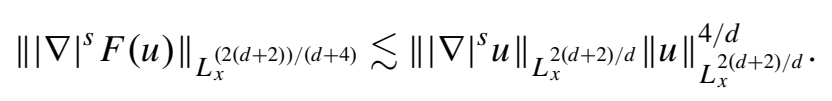

We will need the following sharp Gagliardo-Nirenberg inequality:

Lemma 2.7 [Weinstein 1983]. Let $Q$ be the ground state in the Definition 1.8. Then for any $f \in H_{x}^{1}\left(\mathbb{R}^{d}\right)$, we have

$$
\|f\|_{L_{x}^{2(d+2) / d}}^{2(d+2) / d} \leq \frac{d+2}{d}\left(\frac{M(f)}{M(Q)}\right)^{2 / d}\|\nabla f\|_{L_{x}^{2}}^{2} .
$$

The equality holds only and if only

$$
f=c e^{i \theta} \lambda^{d / 2} Q\left(\lambda\left(x-x_{0}\right)\right)
$$

for $(c, \theta, \lambda) \in\left(\mathbb{R}^{+}, \mathbb{R}, \mathbb{R}^{+}\right)$.

Strichartz estimates. The free Schrödinger flow has the explicit expression

$$
e^{i t \Delta} f(x)=\frac{1}{(4 \pi t)^{d / 2}} \int_{\mathbb{R}^{d}} e^{i|x-y|^{2} / 4 t} f(y) d y,
$$

from which we can derive the kernel estimate of the frequency localized propagator.

Lemma 2.8 (kernel estimates [Killip et al. 2008; 2009b]). For any $m \geq 0$, we have

$$
\mid\left(P_{N} e^{i t \Delta}(x, y) \mid \lesssim_{m} \begin{cases}|t|^{-d / 2} & \text { if }|x-y| \sim N t, \\ \frac{N^{d}}{\left|N^{2} t\right|^{m}\langle N|x-y|\rangle^{m}} & \text { otherwise, }\end{cases}\right.
$$

for $|t| \geq N^{-2}$ and

$$
\left|\left(P_{N} e^{i t \Delta}\right)(x, y)\right| \lesssim m N^{d}\langle N|x-y|\rangle^{-m} \quad \text { for }|t| \leq N^{-2}
$$


We will frequently use the standard Strichartz estimate. Let $d \geq 3$. Let $I$ be a time interval. We define the Strichartz space on $I$ :

$$
S(I)=L_{t}^{\infty} L_{x}^{2}\left(I \times \mathbb{R}^{d}\right) \cap L_{t}^{2} L_{x}^{2 d /(d-2)}\left(I \times \mathbb{R}^{d}\right) .
$$

We also define $N(I)$ to be $L_{t}^{1} L_{x}^{2}\left(I \times \mathbb{R}^{d}\right)+L_{t}^{2} L_{x}^{2 d /(d+2)}\left(I \times \mathbb{R}^{d}\right)$. Then the standard Strichartz estimate reads:

Lemma 2.9 (Strichartz). Let $d \geq 3$. Let I be an interval, $t_{0} \in I$, and let $u_{0} \in L_{x}^{2}\left(\mathbb{R}^{d}\right)$ and $f \in N(I)$. Then, the function $u$ defined by

obeys the estimate

$$
u(t):=e^{i\left(t-t_{0}\right) \Delta} u_{0}-i \int_{t_{0}}^{t} e^{i\left(t-t^{\prime}\right) \Delta} f\left(t^{\prime}\right) d t^{\prime}
$$

$$
\|u\|_{S(I)} \lesssim\left\|u_{0}\right\|_{L_{x}^{2}}+\|f\|_{N(I)},
$$

where all spacetime norms are over $I \times \mathbb{R}^{d}$.

Proof. See, for example, [Ginibre and Velo 1992; Strichartz 1977]. For the endpoint see [Keel and Tao 1998].

We will also need a weighted Strichartz estimate, which exploits heavily the spherical symmetry in order to obtain spatial decay.

Lemma 2.10 (weighted Strichartz [Killip et al. 2008; 2009b]). Let I be an interval, $t_{0} \in I$, and let $F: I \times \mathbb{R}^{d} \rightarrow \mathbb{C}$ be spherically symmetric. Then,

$$
\left\|\int_{t_{0}}^{t} e^{i\left(t-t^{\prime}\right) \Delta} F\left(t^{\prime}\right) d t^{\prime}\right\|_{L_{x}^{2}} \lesssim\left\||x|^{-2(d-1) / q} F\right\|_{L_{t}^{q /(q-1)} L_{x}^{2 q /(q+4)}\left(I \times \mathbb{R}^{d}\right)}
$$

for all $4 \leq q \leq \infty$.

The in/out decomposition. We will need an incoming/outgoing decomposition; we will use the one developed in [Killip et al. 2008; 2009b]. As there, we define operators $P^{ \pm}$by

$$
\left[P^{ \pm} f\right](r):=\frac{1}{2} f(r) \pm \frac{i}{\pi} \int_{0}^{\infty} \frac{r^{2-d} f(\rho) \rho^{d-1} d \rho}{r^{2}-\rho^{2}},
$$

where the radial function $f: \mathbb{R}^{d} \rightarrow \mathbb{C}$ is written as a function of radius only. We will denote by $P^{+}$the projection onto outgoing spherical waves; however, it is not a true projection as it is neither idempotent nor self-adjoint. Similarly, $P^{-}$plays the role of a projection onto incoming spherical waves; its kernel is the complex conjugate of the kernel of $P^{+}$as required by time-reversal symmetry.

For $N>0$ let $P_{N}^{ \pm}$denote the product $P^{ \pm} P_{N}$, where $P_{N}$ is the Littlewood-Paley projection. We record the following properties of $P^{ \pm}$:

Proposition 2.11 (properties of $P^{ \pm}$[Killip et al. 2008; 2009b]).

(i) $P^{+}+P^{-}$represents the projection from $L^{2}$ onto $L_{\mathrm{rad}}^{2}$.

(ii) Fix $N>0$. Then

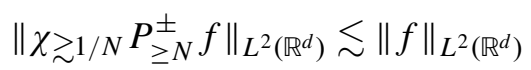

with an $N$-independent constant. 
(iii) If the dimension $d=2$, then $P^{ \pm}$are bounded on $L^{2}\left(\mathbb{R}^{2}\right)$.

(iv) For $|x| \gtrsim N^{-1}$ and $t \gtrsim N^{-2}$, the integral kernel obeys

$$
\left|\left[P_{N}^{ \pm} e^{\mp i t \Delta}\right](x, y)\right| \lesssim \begin{cases}(|x||y|)^{-(d-1) / 2}|t|^{-1 / 2} & |y|-|x| \sim N t, \\ \frac{N^{d}}{(N|x|)^{(d-1) / 2}\langle N|y|\rangle^{(d-1) / 2}}\left\langle N^{2} t+N|x|-N|y|\right\rangle^{-m} & \text { otherwise, }\end{cases}
$$

for all $m \geq 0$.

(v) For $|x| \gtrsim N^{-1}$ and $|t| \lesssim N^{-2}$, the integral kernel obeys

$$
\left|\left[P_{N}^{ \pm} e^{\mp i t \Delta}\right](x, y)\right| \lesssim \frac{N^{d}}{(N|x|)^{(d-1) / 2}\langle N|y|\rangle^{(d-1) / 2}}\left\langle N|x|-\left.N|y|\right|^{-m}\right.
$$

for any $m \geq 0$.

\section{Theorem 1.3 implies Corollary 1.10 and Theorem 1.12}

In this section, we assume Theorem 1.3 holds momentarily and prove the scattering and the rigidity results Corollary 1.10 and Theorem 1.12.

Proof of Corollary 1.10. Suppose by contradiction that Corollary 1.10 does not hold. Then there exists minimal mass $M_{c}$ for which $M_{c}<\infty$ in the defocusing case, $M_{c}<M(Q)$ in the focusing case and maximal-lifespan solution $u(t, x)$ on $I=\left(-T_{*}, T^{*}\right)$ such that

(1) $u$ is spherically symmetric and $M(u)=M_{c}$;

(2) $u$ is almost periodic modulo scaling on $I$.

See for instance [Tao et al. 2008] for this part of the argument which is by now standard. Applying Theorem 1.3, we know that $u \in H_{x}^{1+\varepsilon}$. We now detail the rest of the argument in the focusing case, since the defocusing case is even simpler. By the sharp Gagliardo-Nirenberg inequality and the fact that $M(u)<M(Q)$ we have

$$
\|u(t)\|_{H_{x}^{1}} \lesssim M(u) 1 .
$$

From this and the standard local theory in $H_{x}^{1}$ we know that $u$ exists globally, that is, $T_{*}=T^{*}=\infty$. In this situation, the contradiction will come from the truncated virial and the kinetic energy localization as we explain now. Let $\phi_{\leq R}$ be the smooth cutoff function, we define the truncated virial as

$$
V_{R}(t)=\int \phi_{\leq R}(x)|x|^{2}|u(t, x)|^{2} d x .
$$

Obviously

$$
V_{R}(t) \lesssim R^{2} \quad \text { for all } t \in \mathbb{R} .
$$

On the other hand, we compute the second derivative of virial with respect to $t$; this gives

$$
\partial_{t t} V_{R}(t)=8 E(u)+O\left(\int_{|x|>R}|\nabla u(t, x)|^{2}+|u(t, x)|^{2(d+2) / d}+\frac{1}{R^{2}} \int_{|x|>R}|u(t, x)|^{2} d x\right) .
$$


Since $M(u)<M(Q)$ and $u \in H_{x}^{1}$, from the sharp Gagliardo-Nirenberg inequality (2-2) we have

$$
E(u)>0 .
$$

Now we can use the kinetic energy localization (1-7) and the Gagliardo-Nirenberg inequality to control the $O($ ) term in (3-2) and finally get

$$
\partial_{t t} V_{R}(t) \geq 4 E(u)>0
$$

by taking $R$ sufficiently large. This obviously contradicts (3-1), finishing the proof of Corollary 1.10.

Proof of Theorem 1.12. Let $d \geq 4$ and let $u$ be the solution of (1-1) satisfying the following:

(1) $M(u)=M(Q)$ and $u$ is spherically symmetric.

(2) $u$ does not scatter in both time directions.

By [Killip et al. 2008] or Corollary 1.10, $M(Q)$ is the minimal mass, and the compactness argument in [Keraani 2006; Bégout and Vargas 2007; Tao et al. 2008] shows that $u$ is almost periodic modulo scaling in both time directions. Now we can apply Theorem 1.3 to deduce that $u \in H_{x}^{1}$. Since from Merle's result, the only finite-time blowup solution must be $\operatorname{Pc}(Q)$ up to symmetries and $\operatorname{Pc}(Q)$ scatters in one time direction, we know from condition (2) that $u$ must be a global solution.

From (2-2), this global solution $u$ satisfies $E(u) \geq 0$. Moreover, the same virial argument as in the proof of Corollary 1.10 precludes the case $E(u)>0$, thus $E(u)=0$. From here the coincidence of the solution with solitary wave follows immediately, again by the sharp Gagliardo-Nirenberg inequality.

\section{The proof of Theorem $\mathbf{1 . 3}$}

The proof of Theorem 1.3 proceeds in two steps. In the first step, we prove that away from the origin, the solution has $H_{x}^{1+\varepsilon}$ regularity. Moreover, a similar (but more refined) argument establishes the spatial decay estimate. These two pieces together suffice for us to establish the kinetic localization estimate. However, in this step, the total kinetic energy does not need to be finite.

In the second step, we prove the total kinetic energy is actually finite by controlling the piece near the spatial origin. Thanks to the first step, we only need to consider a single frequency $P_{N} u$ with spatial cutoff $\phi_{\leq 1}$. We can bound this quantity by the Strichartz norm of $P_{N} u$ on a short time interval $[t, t+1 / \sqrt{N}]$. We then establish a recurrent relation for this local Strichartz norm. Iterating the estimates finitely many times then yields the desired bound. More details are given below.

Before proceeding, we remark that in all of the arguments that follow, the only property we use for an almost periodic modulo scaling solution is that it satisfies the improved Duhamel formula. This was first derived in [Tao et al. 2008].

Proposition 4.1 (improved Duhamel formula [Tao et al. 2008]). Let $u$ be the solution of (1-1) and is almost periodic modulo scaling on the time interval I. Then

$$
u(t)=\lim _{T \rightarrow \inf I}-i \int_{T}^{t} e^{i(t-\tau) \Delta} F(u(\tau)) d \tau=\lim _{T \rightarrow \sup I} i \int_{t}^{T} e^{i(t-\tau) \Delta} F(u(\tau)) d \tau .
$$

Here the limit is in weak $L_{x}^{2}$ sense. 
Remark 4.2. As was already mentioned in Remark 1.7, we actually only need the sequential almost periodicity of the solution for the later proof to work. This would imply the following sequence version of improved Duhamel formula:

$$
u(t)=\lim _{n \rightarrow \infty}-i \int_{T_{n}^{-}}^{t} e^{i(t-\tau) \Delta} F(u(\tau)) d \tau=\lim _{n \rightarrow \infty} i \int_{t}^{T_{n}^{+}} e^{i(t-\tau) \Delta} F(u(\tau)) d \tau .
$$

Here again the limit is in weak $L_{x}^{2}$ sense.

In what follows, we shall only assume that

$$
\left.\begin{array}{l}
u \text { is a maximal lifespan solution on } I \\
u \text { is spherically symmetric in space; } \\
u \text { satisfies the improved Duhamel formula (4-1). }
\end{array}\right\}
$$

By time translation invariance and without loss of generality we also assume $[0,1] \subset I$.

Localization for kinetic energy. The purpose of this section is to establish the uniform in time localization of the kinetic energy for solutions satisfying the conditions (4-2). More precisely, we will prove:

Proposition 4.3 (kinetic energy localization). Suppose u satisfies (4-2). Then there exists a function $C(\eta)$ such that

$$
\left\|\phi_{>C(\eta)} \nabla u(t)\right\|_{L_{x}^{2}} \leq \eta \text { for all } \eta>0, t \in I .
$$

As shown in the proof of [Li and Zhang 2009b, Theorems 1.14-1.15, page 31], Proposition 4.3 will follow immediately from the following two propositions which concern the decay of each single frequency.

Proposition 4.4 (frequency decay estimate). Suppose u satisfies (4-2). Let $\varepsilon=(d-1) / d$. Then for any $t \in I$ and $N \geq 1$, we have

$$
\left\|\phi_{>1} P_{N} u(t)\right\|_{L_{x}^{2}} \lesssim N^{-1-\varepsilon} .
$$

Remark 4.5. The decay $N^{-1-(d-1) / d}$ may seem a bit surprising since the exponent $1+(d-1) / d$ is bigger than the regularity of the nonlinearity $1+4 / d$ for dimension $d>5$. However this is not contradictory since in (4-3) we are only considering the part of the solution away from the origin. In this regime the additional regularity of the solution comes from the smoothing effects of the Schrödinger equation and the radial symmetry. On the other hand for the part of the solution near the origin, we only obtain Sobolev regularity $H^{s}$ for some $s<1+4 / d$ (see (4-24)).

Proposition 4.6 (spatial decay estimate). Suppose u satisfies (4-2). Let $N_{0}, N_{1}$ be two dyadic numbers. Then there exist $R_{0}=R_{0}\left(N_{0}, N_{1}\right)$ and $\delta=\delta(d)$ such that for all $R \geq R_{0}, N \in\left[N_{0}, N_{1}\right]$ and $t \in I$, we have

$$
\left\|\phi_{>R} P_{N} u(t)\right\|_{L_{x}^{2}} \lesssim R^{-\delta} .
$$

The proofs of both propositions have been presented, in various forms, in [Killip et al. 2009a; Li and Zhang 2009b]. We sketch the proofs here for the sake of completeness. The proof of Proposition 4.3 will be skipped since it follows directly from Proposition 4.4 and Proposition 4.6. 
Proof of Proposition 4.4. We first use the in/out decomposition and triangle inequality for the bound

$$
\left\|\phi_{>1} P_{N} u(t)\right\|_{2} \leq\left\|\phi_{>1} P_{N}^{+} u(t)\right\|_{2}+\left\|\phi_{>1} P_{N}^{-} u(t)\right\|_{2} .
$$

Since the two terms give the same contribution, we only estimate, for instance, the outgoing piece. For this piece, we use the forward Duhamel formula. Moreover, we will split the integral into different time regimes and introduce the spatial cutoffs. We have

$$
\begin{aligned}
\left\|\phi_{>1} P_{N}^{+} u(t)\right\|_{2} \lesssim & \left\|\phi_{>1} P_{N}^{+} \int_{t}^{\sup I} e^{i(t-s) \Delta} F(u(s)) d s\right\|_{2} \\
& \lesssim\left\|\phi_{>1} P_{N}^{+} \int_{0}^{\sup I-t} e^{-i s \Delta} F(u(t+s)) d \tau\right\|_{2} \\
\lesssim & \left\|\phi_{>1} P_{N}^{+} \int_{0}^{1 / N} e^{-i s \Delta} \phi_{>1 / 2} F(u(t+s)) d s\right\|_{2} \\
& +\left\|\phi_{>1} P_{N}^{+} \int_{0}^{1 / N} e^{-i s \Delta} \phi_{\leq 1 / 2} F(u(t+s)) d s\right\|_{2} \\
& +\left\|\phi_{>1} P_{N}^{+} \int_{1 / N}^{\sup I-t} e^{-i s \Delta} \phi_{>N s / 2} F(u(t+s)) d s\right\|_{2} \\
& +\left\|\phi_{>1} P_{N}^{+} \int_{1 / N}^{\sup I-t} e^{-i s \Delta} \phi_{\leq N s / 2} F(u(t+s)) d s\right\|_{2} .
\end{aligned}
$$

The main contribution comes from (4-4) and (4-6). To estimate (4-4), we drop the bounded operator $\phi_{>1} P_{N}^{+}$and commute the frequency cutoff $\tilde{P}_{N}$ with the spatial cutoff $\phi_{>1}$ (this produces a harmless high order term by the mismatch estimate Lemma 2.3). Thus we have

$$
(4-4) \lesssim\left\|\int_{0}^{1 / N} e^{-i s \Delta} \phi_{>1 / 2} P_{N / 8<\cdots \leq 8 N} F\left(\phi_{>1 / 4} u(t+s)\right) d\right\|_{2}+N^{-10} .
$$

We now use the weighted Strichartz lemma (Lemma 2.10) to estimate the last term:

$$
(4-4) \lesssim\left\|P_{N / 8<\cdots \leq 8 N} F\left(\phi_{>1 / 4} u(t+s)\right)\right\|_{L_{s}^{d /(d-1)} L_{x}^{2 d /(d+4)}([0,1 / N])}+N^{-10} \lesssim N^{-(d-1) / d} .
$$

The estimate of (4-6) follows in a similar way. Applying the mismatch estimate and weighted Strichartz inequality, we have

$$
\begin{aligned}
(4-6) & \lesssim\left\|\int_{1 / N}^{\sup I-t} e^{-i s \Delta} \phi_{>N s / 2} P_{N / 8<\cdots \leq 8 N} F\left(\phi_{>N s / 4} u(t+s)\right) d s\right\|_{2}+N^{-10} \\
& \lesssim\left\|(N s)^{-2(d-1) / d} P_{N / 8<\cdots \leq 8 N} F\left(\phi_{>N s / 4} u(t+s)\right)\right\|_{L_{s}^{d /(d-1)} L_{x}^{2 d /(d+4)}([1 / N, \sup I-t))}+N^{-10} \\
& \lesssim N^{-2(d-1) / d}\left\|s^{-2(d-1) / d}\right\| F\left(\phi_{>N s / 4} u(t+s)\right) \|_{L_{x}^{2 d /(d+4)} \|_{L_{s}^{d /(d-1)}([1 / N, \sup I-t))}+N^{-10}} \\
& \lesssim N^{-(d-1) / d} .
\end{aligned}
$$


Finally we consider the contribution from the tail terms (4-7) and (4-5). Applying Proposition 2.11, we bound the kernel as follows:

$$
\begin{aligned}
\left|\left(\phi_{>1} P_{N}^{+} e^{-i s \Delta} \phi_{\leq 1 / 2}\right)(x, y)\right| \lesssim N^{-9 d}\langle N(x-y)\rangle^{-10 d} & \text { for } 0<s \leq \frac{1}{N}, \\
\left|\left(\phi_{>1} P_{N}^{+} e^{-i s \Delta} \phi_{\leq N s / 2}\right)(x, y)\right| \lesssim N^{d}\left\langle N^{2} s\right\rangle^{-10 d}\langle N(x-y)\rangle^{-10 d} \lesssim N^{-9 d}\langle N(x-y)\rangle^{-10 d} & \text { for } s>\frac{1}{N} .
\end{aligned}
$$

The desired decay then follows from the kernel estimate and a simple use of Young's inequality. Combining the estimates of these four pieces together, we obtain

$$
\left\|\phi_{>1} P_{N} u(t)\right\|_{L_{x}^{2}} \lesssim N^{-(d-1) / d} \quad \text { for all } t \in I .
$$

Moreover it is easy to check that, after notational change, the same analysis establishes

$$
\left\|\phi_{>c} P_{N} u(t)\right\|_{L_{x}^{2}} \lesssim{ }_{c} N^{-(d-1) / d} \quad \text { for all } t \in I
$$

This implies

$$
\left\||\nabla|^{(d-1) / d-}\left(\phi_{>c} u(t)\right)\right\|_{L_{x}^{2}} \lesssim_{c} 1 \text { for all } t \in I .
$$

Now we can upgrade the decay (4-9) by inserting (4-10) when we repeat the same argument as above. For example, using Bernstein and (4-10), the term (4-4) can be re-estimated as follows:

$$
\begin{aligned}
(4-4) & \lesssim\left\|P_{N / 8<\cdots \leq 8 N} F\left(\phi_{>1 / 4} u(t+s)\right)\right\|_{L_{s}^{d /(d-1)} L_{x}^{2 d /(d+4)}([0,1 / N])} \\
& \lesssim N^{-2 d /(d-1)+}\left\||\nabla|^{(d-1) / d-} F\left(\phi_{>1 / 4} u(t+s)\right)\right\|_{L_{s}^{\infty} L_{x}^{2 d /(d+4)}([0,1 / N])} \\
& \lesssim N^{-2(d-1) / d+} .
\end{aligned}
$$

The same computation applies to (4-6), so we get

$$
\left\|\phi_{>c} P_{N} u(t)\right\|_{2} \lesssim c N^{-2(d-1) / d+} \text { for all } t \in I .
$$

Another repetition of the argument yields (4-3) for $\varepsilon=(d-1) / d$.

The proof of Proposition 4.6 has the same spirit as the proof of Proposition 4.4. So here we only briefly sketch the proof.

Proof sketch of Proposition 4.6. Using the in/out decomposition, it suffices to consider the piece

$$
\left\|\phi_{>R} P_{N}^{+} u(t)\right\|_{2}
$$

for which we use the forward Duhamel formula to express $u(t)$. This further reduces our consideration to the integral

$$
\left\|\phi_{>R} P_{N}^{+} \int_{0}^{\sup I-t} e^{-i s \Delta} F(u(t+s)) d s\right\|_{2} .
$$

Now we split the time integral into regimes where $0<s<R /(100 N)$, and $s>R /(100 N)$. For the small time regime, we insert the spatial cutoff $\phi_{>R / 2}$ and $\phi_{\leq R / 2}$. For the large time regime, we insert the spatial cutoff $\phi_{>N s / 2}$ and $\phi_{\leq N s / 2}$. As indicated in the proof of Proposition 4.4, the pieces with cutoff near the origin will give arbitrary decay in $R$ by using the kernel estimate Proposition 2.11. The pieces with cutoff away from the origin can be dealt with by the weighted Strichartz estimate. The point here 
is that since the frequencies are fixed in the dyadic interval $\left[N_{0}, N_{1}\right]$, we can take $R$ sufficiently large to cancel any $N$ dependent quantity.

Local iteration to prove $\boldsymbol{H}_{\boldsymbol{x}}^{\mathbf{1 +}}$ regularity. In this part, we prove $u(0)=u_{0} \in H_{x}^{1+}$. This amounts to showing $\left\|P_{\geq N} u_{0}\right\|_{L_{x}^{2}} \lesssim N^{-1-}$ for $N \geq 1$. Using Proposition 4.4, we first show the quantity $\left\|P_{\geq N} u_{0}\right\|_{L_{x}^{2}}$ is determined by the dual Strichartz norm of the nonlinearity on the local time interval $[0,1 / \sqrt{N}]$.

Lemma 4.7. Let $u$ satisfy (4-2). Let $\varepsilon=(d-1) / d$. Then for any $N \geq 1$, we have

$$
\left\|P_{\geq N} u_{0}\right\|_{L_{x}^{2}} \leq C\left(d,\left\|u_{0}\right\|_{L_{x}^{2}}\right)\left(N^{-1-\varepsilon}+\left\|P_{\geq N} F(u)\right\|_{L_{t, x}^{2(d+2) /(d+4)}\left([0,1 / \sqrt{N}] \times \mathbb{R}^{d}\right)}\right) .
$$

Remark 4.8. Here the choice of the time interval cutoff at $N^{-1 / 2}$ is not special. Perhaps a more natural choice is $1 / N$ since the solution propagates at speed $N$ and one is localizing to spacial scale $O(1)$. This latter choice would also work for our iteration scheme.

Proof. Since by Proposition 4.4, we have that $\left\|\phi_{>1} P_{\geq N} u_{0}\right\|_{L_{x}^{2}} \lesssim N^{-1-\varepsilon}$, we only need to estimate the piece $\left\|\phi_{\leq 1} P_{\geq N} u_{0}\right\|_{L_{x}^{2}}$. In the following, the implicit constants are allowed to depend on $d$ and $\left\|u_{0}\right\|_{L_{x}^{2}}$. By the improved Duhamel formula we get

$$
\begin{aligned}
\left\|\phi_{\leq 1} P_{\geq N} u_{0}\right\|_{L_{x}^{2}} \leq & \left\|\phi_{\leq 1} P_{\geq N} \int_{0}^{\sup I} e^{-i \tau \Delta} F(u(\tau)) d \tau\right\|_{L_{x}^{2}} \\
\leq & \left\|\phi_{\leq 1} P_{\geq N} \int_{0}^{1 / \sqrt{N}} e^{-i \tau \Delta} F(u(\tau)) d \tau\right\|_{L_{x}^{2}} \\
& +\left\|\phi_{\leq 1} P_{\geq N} \int_{1 / \sqrt{N}}^{\sup I} e^{-i \tau \Delta} \phi_{\leq N \tau / 8} F(u(\tau)) d \tau\right\|_{L_{x}^{2}} \\
& +\left\|\phi_{\leq 1} P_{\geq N} \int_{1 / \sqrt{N}}^{\sup I} e^{-i \tau \Delta} \phi_{>N \tau / 8} F(u(\tau)) d \tau\right\|_{L_{x}^{2}} .
\end{aligned}
$$

For (4-12), we use Strichartz to bound it by

$$
\left\|P_{\geq N} F(u)\right\|_{L_{t, x}^{2(d+2) /(d+4)}\left([0,1 / \sqrt{N}] \times \mathbb{R}^{d}\right)} .
$$

For (4-13), using the kernel estimate with $m=10 d$, we have

$$
\begin{aligned}
(4-13) & \leq \sum_{M \geq N}\left\|\phi_{\leq 1} P_{M} \int_{1 / \sqrt{N}}^{\sup I} e^{-i \tau \Delta} \phi_{\leq N \tau / 8} F(u(\tau)) d \tau\right\|_{L_{x}^{2}} \\
& \lesssim \sum_{M \geq N} M^{d-20 d} \int_{1 / \sqrt{N}}^{\sup I} \tau^{-10 d}\left\|\langle M|\cdot|\rangle^{-10 d} * F(u)\right\|_{L_{x}^{2}} d \tau \\
& \lesssim \sum_{M \geq N} M^{d-20 d} M^{(1 / 2)(10 d-1)}\|F(u)\|_{L_{\tau}^{\infty} L_{x}^{2 d /(d+4)}\left\|\langle M|\cdot|\rangle^{-10 d}\right\|_{L_{x}^{d /(d-2)}}} \\
& \lesssim \sum_{M \geq N} M^{(3 / 2)(1-10 d)} \\
& \lesssim N^{-10} .
\end{aligned}
$$


For (4-14), by the triangle inequality, we have

$$
\begin{aligned}
(4-14) \lesssim & \left\|P_{\geq N} \int_{1 / \sqrt{N}}^{\sup I} e^{-i \tau \Delta} \phi_{>N \tau / 8} F\left(u \phi_{>1 / 8}\right)(\tau) d \tau\right\|_{L_{x}^{2}} \\
\lesssim & \left\|P_{\geq N} \int_{1 / \sqrt{N}}^{\sup I} e^{-i \tau \Delta} \phi_{>N \tau / 8} P_{\leq N / 8} F\left(u \phi_{>1 / 8}\right)(\tau) d \tau\right\|_{L_{x}^{2}} \\
& +\left\|P_{\geq N} \int_{1 / \sqrt{N}}^{\sup I} e^{-i \tau \Delta} \phi_{>N \tau / 8} P_{>N / 8} F\left(u \phi_{>1 / 8}\right)(\tau) d \tau\right\|_{L_{x}^{2}}
\end{aligned}
$$

For the term (4-15), we use the mismatch estimate Lemma 2.3 and Bernstein to bound it as

$$
(4-15) \lesssim \int_{1 / \sqrt{N}}^{\sup I}\left(N^{2} \tau\right)^{-10 d}\left\|P_{\leq N / 8} F\left(u \phi_{>1 / 8}\right)\right\|_{L_{x}^{2}} d \tau \lesssim \int_{1 / \sqrt{N}}^{\sup I}\left(N^{2} \tau\right)^{-10 d} N^{2} d \tau \lesssim N^{-5} .
$$

For the term (4-16), we use weighted Strichartz to estimate and Proposition 4.3 to get

$$
\begin{aligned}
(4-16) & \lesssim\left\|(N \tau)^{-2(d-1) / d} P_{>N / 8} F\left(u \phi_{>1 / 8}\right)\right\|_{L_{\tau}^{d /(d-1)} L_{x}^{2 d /(d+4)}\left([1 / \sqrt{N}, \sup I) \times \mathbb{R}^{d}\right)} \\
& \lesssim N^{-2(d-1) / d}\left\|\tau^{-2(d-1) / d}\right\|_{L_{\tau}^{d /(d-1)}([1 / \sqrt{N}, \sup I))} \cdot N^{-1}\left\|\nabla P_{>N / 8} F\left(u \phi_{>1 / 8}\right)\right\|_{L_{\tau}^{\infty} L_{x}^{2 d /(d+4)}} \\
& \lesssim N^{-1-3(d-1) /(2 d)} .
\end{aligned}
$$

This finishes the proof of Lemma 4.7.

Now we further estimate the dual Strichartz norm of the nonlinearity.

Lemma 4.9 (dual Strichartz norm control). Let u satisfy (4-2). Let $\beta>0, N_{0} \geq 1, N>(1 / \beta) N_{0}$. Then for any $0<s<1+4 / d$, we have

$$
\begin{aligned}
& \left\|P_{\geq N} F(u)\right\|_{L_{t, x}^{2(d+2) /(d+4)}\left([0,1 / \sqrt{N}] \times \mathbb{R}^{d}\right)} \\
& \lesssim\|u\|_{S([0,1 / \sqrt{N}])}^{4 / d} \sum_{M \leq \beta N}\left(\frac{M}{N}\right)^{S}\left\|P_{M} u\right\|_{S([0,1 / \sqrt{N}])} \\
& +\left\|u_{>\beta N}\right\|_{S([0,1 / \sqrt{N}])}\left(N_{0}^{4 /(d+2)} N^{-1 /(d+2)}+\left\|u_{>N_{0}}\right\|_{L_{\tau}^{\infty} L_{x}^{2}}^{8 /(d(d+2))}\left\|u_{>N_{0}}\right\|_{S([0,1 / \sqrt{N}])}^{4 /(d+2)}\right) .
\end{aligned}
$$

Proof. By splitting $u$ into low, medium and high frequencies, $u=u_{\leq N_{0}}+u_{N_{0}<\cdots \leq \beta N}+u_{>\beta N}$, we write

$$
F(u)=F\left(u_{\leq \beta N}\right)+O\left(u_{>\beta N}\left|u_{\leq N_{0}}\right|^{4 / d}\right)+O\left(u_{>\beta N}\left|u_{>N_{0}}\right|^{4 / d}\right) .
$$

The contribution due to the first term can be estimated as follows. By using Lemma 2.6, we have $\left\|P_{\geq N} F\left(u_{\leq \beta N}\right)\right\|_{L_{t, x}^{2(d+2) /(d+4)}\left([0,1 / \sqrt{N}] \times \mathbb{R}^{d}\right)}$

$$
\begin{aligned}
& \lesssim N^{-s}\left\||\nabla|^{s} P_{\geq N} F\left(u_{\leq \beta N}\right)\right\|_{L_{t, x}^{2(d+2) /(d+4)}\left([0,1 / \sqrt{N}] \times \mathbb{R}^{d}\right)} \\
& \lesssim N^{-s}\left\||\nabla|^{s} u_{\leq \beta N}\right\|_{L_{t, x}^{2(d+2) / d}\left([0,1 / \sqrt{N}] \times \mathbb{R}^{d}\right)}\left\|u_{\leq \beta N}\right\|_{L_{t, x}^{2 / d}}^{2 / d+2) / d}\left([0,1 / \sqrt{N}] \times \mathbb{R}^{d}\right) \\
& \lesssim\|u\|_{S([0,1 / \sqrt{N}])}^{4 / d} \sum_{M \leq \beta N}\left(\frac{M}{N}\right)^{s}\left\|P_{M} u\right\|_{S([0,1 / \sqrt{N}])} .
\end{aligned}
$$


For the contribution due to the second part of (4-18), we use Bernstein to get

$$
\begin{aligned}
\left\|u_{>\beta N}\left|u_{\leq N_{0}}\right|^{4 / d}\right\|_{L_{t, x}^{2(d+2) /(d+4)}\left([0,1 / \sqrt{N}] \times \mathbb{R}^{d}\right)} & \lesssim\left\|u_{>\beta N}\right\|_{L_{t, x}^{2(d+2) / d}\left([0,1 / \sqrt{N}] \times \mathbb{R}^{d}\right)}\left\|u_{\leq N_{0}}\right\|_{L_{t, x}^{2(d+2) / d}\left([0,1 / \sqrt{N}] \times \mathbb{R}^{d}\right)}^{4 / d} \\
& \lesssim\left\|u_{>\beta N}\right\|_{S([0,1 / \sqrt{N}])} N_{0}^{4 /(d+2)} N^{-1 /(d+2)}\left\|u_{\leq N_{0}}\right\|_{L_{\tau}^{\infty} L_{x}^{2}}^{4 / d} \\
& \lesssim\left\|u_{>\beta N}\right\|_{S([0,1 / \sqrt{N}])} N_{0}^{4 /(d+2)} N^{-1 /(d+2)} .
\end{aligned}
$$

For the third term in (4-18), we use Hölder and interpolation to get

$\left\|u_{>\beta N}\left|u_{>N_{0}}\right|^{4 / d}\right\|_{L_{t, x}^{2(d+2) /(d+4)}\left([0,1 / \sqrt{N}] \times \mathbb{R}^{d}\right)}$

$$
\begin{aligned}
& \lesssim\left\|u_{>\beta N}\right\|_{L_{t, x}^{2(d+2) / d}\left([0,1 / \sqrt{N}] \times \mathbb{R}^{d}\right)}\left\|u_{>N_{0}}\right\|_{L_{t, x}^{2(d+2) / d}\left([0,1 / \sqrt{N}] \times \mathbb{R}^{d}\right)}^{4 / d} \\
& \lesssim\left\|u_{>\beta N}\right\|_{S([0,1 / \sqrt{N}])}\left\|u_{>N_{0}}\right\|_{L_{t}^{\infty} L_{x}^{2}\left([0,1 / \sqrt{N}] \times \mathbb{R}^{d}\right)}^{8 /((d+2)}\left\|u_{>N_{0}}\right\|_{S([0,1 / \sqrt{N}])}^{4 /(d+2)}
\end{aligned}
$$

Collecting the three pieces together, we get (4-17).

Now by Strichartz estimate,

$$
\left\|P_{\geq N} u\right\|_{S([0,1 / \sqrt{N}])} \lesssim\left\|P_{\geq N} u_{0}\right\|_{L_{x}^{2}}+\left\|P_{\geq N} F(u)\right\|_{L_{t, x}^{2(d+2) /(d+4)}\left([0,1 / \sqrt{N}] \times \mathbb{R}^{d}\right)},
$$

and the latter is in turn determined by $\left\|P_{\geq N} u\right\|_{S([0,1 / \sqrt{N}])}$ due to Lemma 4.7 and Lemma 4.9. This enables us to set up a recurrent relation for $\left\|P_{\geq N} u\right\|_{S([0,1 / \sqrt{N}])}$.

We define

$$
A_{N}=\left\|P_{\geq N} u\right\|_{S([0,1 / \sqrt{N}])} .
$$

Since locally the Strichartz norm of $u$ is bounded, we can write

$$
A:=\|u\|_{S([0,1])}+1<\infty .
$$

Using the Strichartz inequality, Lemma 4.7, Lemma 4.9 and taking $s=1+2 / d$, we obtain

$$
\begin{aligned}
A_{N} \leq C(d)\left(\left\|P_{\geq N} u_{0}\right\|_{L_{x}^{2}}+\left\|P_{\geq N} F(u)\right\|_{L_{t, x}^{2(d+2) /(d+4)}\left([0,1 / \sqrt{N}] \times \mathbb{R}^{d}\right)}\right) \\
\leq C\left(d,\left\|u_{0}\right\|_{L_{x}^{2}}\right)\left(N^{-1-\varepsilon}\right. \\
\quad+A^{4 / d} \sum_{M \leq \beta N}\left(\frac{M}{N}\right)^{1+2 / d}\left\|P_{M} u\right\|_{S([0,1 / \sqrt{N}])} \\
\quad+\left\|P_{\geq \beta N} u\right\|_{S([0,1 / \sqrt{N}])}\left(N_{0}^{4 /(d+2)} N^{-1 /(d+2)}+A^{4 /(d+2)}\left\|u_{\geq N_{0}}\right\|_{L_{t}^{\infty} L_{x}^{2}([0,1 / \sqrt{N}])}^{8 /(d(d))}\right) .
\end{aligned}
$$

For (4-19), we do a little modification. Noting $P_{M}=P_{M} P_{\geq M / 2}$, we have

$$
\begin{aligned}
\text { (4-19) } & \lesssim A^{4 / d} \sum_{M \leq \beta N}\left(\frac{M}{N}\right)^{1+2 / d}\left\|P_{\geq M / 2} u\right\|_{S([0,1 / \sqrt{N}])} \\
& \lesssim A^{4 / d} \sum_{M \leq 2 \beta N}\left(\frac{M}{N}\right)^{1+2 / d}\left\|P_{\geq M} u\right\|_{S([0,1 / \sqrt{N}])} .
\end{aligned}
$$

We shall take $\beta$ to be sufficiently small. The constraint on $\beta$ will be specified later. 
Now we absorb (4-20) into (4-19) through taking suitable parameters. First we take $N_{0}=N_{0}(\beta, A)$ such that

$$
A^{4 /(d+2)}\left\|u_{>N_{0}}\right\|_{L_{t}^{\infty} L_{x}^{2}([0,1])}^{8 /(d(d+2))} \leq \frac{1}{100} \beta^{1+2 / d} .
$$

This is certainly possible since $u \in C\left([0,1], L_{x}^{2}\right)$ and $[0,1]$ is a compact interval. Then we assume $N \geq M_{0}$ where

$$
M_{0}^{-1 /(d+2)} N_{0}^{4 /(d+2)} \leq \frac{1}{100} \beta^{1+2 / d} .
$$

Under these restrictions we have

$$
(4-20) \leq \frac{1}{2} \beta^{1+2 / d}\left\|P_{\geq \beta N} u\right\|_{S([0,1 / \sqrt{N}])} .
$$

Therefore we get for all $N \geq M_{0}$ that

$$
\begin{aligned}
A_{N} & \leq C\left(d,\left\|u_{0}\right\|_{L_{x}^{2}}\right)\left(N^{-1-\varepsilon}+\sum_{M \leq 2 \beta N}\left(\frac{M}{N}\right)^{1+2 / d}\left\|P_{\geq M} u\right\|_{S([0,1 / \sqrt{N}])}\right) \\
& \leq N^{-1-\varepsilon / 2}+\sum_{M \leq 2 \beta N}\left(\frac{M}{N}\right)^{1+1 / d}\left\|P_{\geq M} u\right\|_{S([0,1 / \sqrt{N}])},
\end{aligned}
$$

where in the last inequality we have killed the constant $C\left(d,\left\|u_{0}\right\|_{L_{x}^{2}}\right)$. This is possible by first taking $\beta$ sufficiently small, then taking $M_{0}$ large enough.

We split the summation into $M \leq M_{0}$ and $M>M_{0}$. For large $M$, we trivially bound the summand by

$$
\left(\frac{M}{N}\right)^{1+1 / d} A_{M}
$$

Then we sum all the pieces for small $M$, which gives that

$$
\sum_{M \leq M_{0}}\left(\frac{M}{N}\right)^{1+1 / d}\left\|P_{\geq M} u\right\|_{S([0,1 / \sqrt{N}])} \lesssim A M_{0}^{1+1 / d} N^{-1-1 / d} .
$$

Finally we establish the following recurrence relation for $A_{N}$ : Let $s=1 / d+1$. Then there exists $C_{1}>0$ such that for all $N \geq M_{0}$,

$$
A_{N} \leq C_{1} M_{0}^{s} N^{-s}+\sum_{M_{0}<M \leq 2 \beta N}\left(\frac{M}{N}\right)^{s} A_{M} .
$$

This combined with the trivial bound $A_{N} \leq A$ will give us the final control on $A_{N}$,

$$
A_{N} \leq C\left(A, M_{0}\right) N^{-s+} \text { for all } N \geq M_{0}
$$

if we apply the following lemma:

Lemma 4.10 (recursive control). Let $s>1, \gamma>0$ and $s-\gamma>1$. Let $C_{1}>0$ be such that for all $N \geq M_{0}$,

$$
\begin{aligned}
& A_{N} \leq C_{1} M_{0}^{s} N^{-s}+\sum_{M_{0} \leq M \leq \beta^{\prime} N}\left(\frac{M}{N}\right)^{s} A_{M}, \\
& A_{N} \leq A .
\end{aligned}
$$


Then there exists a constant $c(s, \gamma, A)>0$ such that for all $0<\beta^{\prime}<c(s, \gamma, A)$, we have

$$
A_{N} \leq 2 C_{1} M_{0}^{s} N^{-s+\gamma} \quad \text { for all } N \geq M_{0} .
$$

Proof. We will inductively prove that

$$
A_{N} \leq 2 C_{1} M_{0}^{s} N^{-s+\gamma}+\left(\beta^{\prime}\right)^{j}
$$

First, plugging the bound (4-26) into (4-25), we get

$$
A_{N} \leq C_{1} M_{0} N^{-s}+C(s) A\left(\beta^{\prime}\right)^{s} \leq 2 C_{1} M_{0} N^{-s+\gamma}+\beta^{\prime},
$$

by requiring $\left(\beta^{\prime}\right)^{s-1}<1 /(100 C(s) A)$. This establishes (4-28) for $j=1$.

Now assuming (4-28) holds for $j$-th step, we plug this bound into (4-25) to compute

$$
\begin{aligned}
A_{N} & \leq C_{1} M_{0}^{s} N^{-s}+2 C(s)\left(\beta^{\prime}\right)^{\gamma} \cdot C_{1} M_{0}^{s} N^{-s+\gamma}+C(s)\left(\beta^{\prime}\right)^{s-1} \cdot\left(\beta^{\prime}\right)^{j+1} \\
& \leq 2 C_{1} M_{0}^{s} N^{-s+\gamma}+\left(\beta^{\prime}\right)^{j+1},
\end{aligned}
$$

by requiring $\left(\beta^{\prime}\right)^{\gamma}<1 /(100 C(s))$. This establishes (4-28) for $j+1$.

Finally, (4-27) follows by taking $j \rightarrow \infty$ in (4-28).

\section{Acknowledgements}

Both authors were supported by start-up funding from the Mathematics Department of the University of Iowa. Li was also supported by NSF grant DMS-0908032 and an Old Gold summer fellowship from the University of Iowa. Zhang was also supported by an Alfred P. Sloan Research Fellowship and Project 973 in China.

\section{References}

[Bégout and Vargas 2007] P. Bégout and A. Vargas, "Mass concentration phenomena for the $L^{2}$-critical nonlinear Schrödinger equation", Trans. Amer. Math. Soc. 359:11 (2007), 5257-5282. MR 2008g:35190

[Berestycki and Lions 1979] H. Berestycki and P.-L. Lions, "Existence d'ondes solitaires dans des problèmes nonlinéaires du type Klein-Gordon”, C. R. Acad. Sci. Paris Sér. A-B 288:7 (1979), A395-A398. MR 80i:35076 Zbl 0397.35024

[Cazenave 2003] T. Cazenave, Semilinear Schrödinger equations, Courant Lecture Notes in Math. 10, NYU Courant Inst. of Math. Sciences, New York, 2003. MR 2004j:35266 Zbl 1055.35003

[Christ and Weinstein 1991] F. M. Christ and M. I. Weinstein, "Dispersion of small amplitude solutions of the generalized Korteweg-de Vries equation”, J. Funct. Anal. 100:1 (1991), 87-109. MR 92h:35203 Zbl 0743.35067

[Ginibre and Velo 1992] J. Ginibre and G. Velo, "Smoothing properties and retarded estimates for some dispersive evolution equations", Comm. Math. Phys. 144:1 (1992), 163-188. MR 93a:35065 Zbl 0762.35008

[Glassey 1977] R. T. Glassey, "On the blowing up of solutions to the Cauchy problem for nonlinear Schrödinger equations", $J$. Math. Phys. 18:9 (1977), 1794-1797. MR 57 \#842

[Hmidi and Keraani 2005] T. Hmidi and S. Keraani, "Blowup theory for the critical nonlinear Schrödinger equations revisited", Int. Math. Res. Not. 46 (2005), 2815-2828. MR 2007k:35464 Zbl 1126.35067

[Keel and Tao 1998] M. Keel and T. Tao, "Endpoint Strichartz estimates", Amer. J. Math. 120:5 (1998), 955-980. MR 2000d: 35018 Zbl 0922.35028

[Kenig and Merle 2006] C. E. Kenig and F. Merle, "Global well-posedness, scattering and blow-up for the energy-critical, focusing, non-linear Schrödinger equation in the radial case", Invent. Math. 166:3 (2006), 645-675. MR 2007g:35232 Zbl 1115.35125 
[Keraani 2001] S. Keraani, "On the defect of compactness for the Strichartz estimates of the Schrödinger equations", J. Differential Equations 175:2 (2001), 353-392. MR 2002j:35281 Zbl 1038.35119

[Keraani 2006] S. Keraani, "On the blow up phenomenon of the critical nonlinear Schrödinger equation”, J. Funct. Anal. 235:1 (2006), 171-192. MR 2007e:35260 Zbl 1099.35132

[Killip et al. 2008] R. Killip, M. Visan, and X. Zhang, "The mass-critical nonlinear Schrödinger equation with radial data in dimensions three and higher", Anal. PDE 1:2 (2008), 229-266. MR MR2472890 Zbl 1171.35111

[Killip et al. 2009a] R. Killip, D. Li, M. Visan, and X. Zhang, "Characterization of minimal-mass blowup solutions to the focusing mass-critical NLS”, SIAM J. Math. Anal. 41:1 (2009), 219-236. MR MR2505858

[Killip et al. 2009b] R. Killip, T. Tao, and M. Visan, "The cubic nonlinear Schrödinger equation in two dimensions with radial data”, J. Eur. Math. Soc. 11:6 (2009), 1203-1258. MR MR2557134 Zbl 05641373

[Kwong 1989] M. K. Kwong, "Uniqueness of positive solutions of $\Delta u-u+u^{p}=0$ in $\mathbf{R}^{n}$ ", Arch. Rational Mech. Anal. 105:3 (1989), 243-266. MR 90d:35015 Zbl 0676.35032

[Li and Zhang 2009a] D. Li and X. Zhang, "On the focusing mass critical problem in six dimensions with splitting spherically symmetric initial data", preprint, 2009. Submitted to Discrete Contin. Dynam. Systems.

[Li and Zhang 2009b] D. Li and X. Zhang, "On the rigidity of solitary waves for the focusing mass-critical NLS in dimensions $d \geq 2$ ", preprint, 2009. arXiv 0902.0802

[Merle 1993] F. Merle, "Determination of blow-up solutions with minimal mass for nonlinear Schrödinger equations with critical power", Duke Math. J. 69:2 (1993), 427-454. MR 94b:35262 Zbl 0808.35141

[Merle and Raphael 2005] F. Merle and P. Raphael, "The blow-up dynamic and upper bound on the blow-up rate for critical nonlinear Schrödinger equation”, Ann. of Math. (2) 161:1 (2005), 157-222. MR 2006k:35277 Zbl 02204253

[Staffilani 1997] G. Staffilani, "On the generalized Korteweg-de Vries-type equations", Differential Integral Equations 10:4 (1997), 777-796. MR 2001a:35005 Zbl 0891.35135

[Strichartz 1977] R. S. Strichartz, "Restrictions of Fourier transforms to quadratic surfaces and decay of solutions of wave equations", Duke Math. J. 44:3 (1977), 705-714. MR 58 \#23577 Zbl 0372.35001

[Tao et al. 2007] T. Tao, M. Visan, and X. Zhang, "Global well-posedness and scattering for the defocusing mass-critical nonlinear Schrödinger equation for radial data in high dimensions", Duke Math. J. 140:1 (2007), 165-202. MR 2010a:35249 Zbl 05208403

[Tao et al. 2008] T. Tao, M. Visan, and X. Zhang, "Minimal-mass blowup solutions of the mass-critical NLS", Forum Math. 20:5 (2008), 881-919. MR 2009m:35495 Zbl 1154.35085

[Taylor 2000] M. E. Taylor, Tools for PDE. Pseudodifferential operators, paradifferential operators, and layer potentials, Math. Surveys and Monogr. 81, Amer. Math. Soc., Providence, RI, 2000. MR 2001g:35004 Zbl 0963.35211

[Weinstein 1983] M. I. Weinstein, "Nonlinear Schrödinger equations and sharp interpolation estimates", Comm. Math. Phys. 87:4 (1983), 567-576. MR 84d:35140 Zbl 0527.35023

[Weinstein 1986] M. I. Weinstein, "On the structure and formation of singularities in solutions to nonlinear dispersive evolution equations”, Comm. Partial Differential Equations 11:5 (1986), 545-565. MR 87i:35026 Zbl 0596.35022

Received 10 Aug 2009. Revised 18 Nov 2009. Accepted 17 Dec 2009.

DoNG LI: mpdongli@gmail.com

Department of Mathematics, University of Iowa, 14 MacLean Hall, Iowa City, IA 52240, United States

XIAOYI ZHANG: zh.xiaoyi@gmail.com

Academy of Mathematics and System Sciences, Beijing, China

and

Department of Mathematics, University of Iowa, 14 MacLean Hall, Iowa City, IA 52240, United States 


\title{
Analysis \& PDE
}

pjm.math.berkeley.edu/apde

EDITORS

\author{
EDITOR-IN-CHIEF \\ Maciej Zworski \\ University of California \\ Berkeley, USA
}

BOARD OF EDITORS

\begin{tabular}{|c|c|c|c|}
\hline Michael Aizenman & $\begin{array}{l}\text { Princeton University, USA } \\
\text { aizenman@math.princeton.edu }\end{array}$ & Nicolas Burq & $\begin{array}{l}\text { Université Paris-Sud 11, France } \\
\text { nicolas.burq@math.u-psud.fr }\end{array}$ \\
\hline Luis A. Caffarelli & $\begin{array}{l}\text { University of Texas, USA } \\
\text { caffarel@math.utexas.edu }\end{array}$ & un-Yung Alice Chang & $\begin{array}{l}\text { Princeton University, USA } \\
\text { chang@math.princeton.edu }\end{array}$ \\
\hline Michael Christ & $\begin{array}{l}\text { University of California, Berkeley, USA } \\
\text { mchrist@math.berkeley.edu }\end{array}$ & Charles Fefferman & $\begin{array}{l}\text { Princeton University, USA } \\
\text { cf@math.princeton.edu }\end{array}$ \\
\hline Ursula Hamenstaedt & $\begin{array}{l}\text { Universität Bonn, Germany } \\
\text { ursula@math.uni-bonn.de }\end{array}$ & Nigel Higson & $\begin{array}{l}\text { Pennsylvania State Univesity, USA } \\
\text { higson@math.psu.edu }\end{array}$ \\
\hline Vaughan Jones & $\begin{array}{l}\text { University of California, Berkeley, USA } \\
\text { vfr@math.berkeley.edu }\end{array}$ & Herbert Koch & $\begin{array}{l}\text { Universität Bonn, Germany } \\
\text { koch@math.uni-bonn.de }\end{array}$ \\
\hline Izabella Laba & $\begin{array}{l}\text { University of British Columbia, Canada } \\
\text { ilaba@math.ubc.ca }\end{array}$ & Gilles Lebeau & $\begin{array}{l}\text { Université de Nice Sophia Antipolis, France } \\
\text { lebeau@unice.fr }\end{array}$ \\
\hline László Lempert & $\begin{array}{l}\text { Purdue University, USA } \\
\text { lempert@math.purdue.edu }\end{array}$ & Richard B. Melrose & $\begin{array}{l}\text { Massachussets Institute of Technology, USA } \\
\text { rbm@math.mit.edu }\end{array}$ \\
\hline Frank Merle & $\begin{array}{l}\text { Université de Cergy-Pontoise, France } \\
\text { Frank.Merle@u-cergy.fr }\end{array}$ & William Minicozzi II & $\begin{array}{l}\text { Johns Hopkins University, USA } \\
\text { minicozz@math.jhu.edu }\end{array}$ \\
\hline Werner Müller & $\begin{array}{l}\text { Universität Bonn, Germany } \\
\text { mueller@math.uni-bonn.de }\end{array}$ & Yuval Peres & $\begin{array}{l}\text { University of California, Berkeley, USA } \\
\text { peres@stat.berkeley.edu }\end{array}$ \\
\hline Gilles Pisier & $\begin{array}{l}\text { Texas A\&M University, and Paris } 6 \\
\text { pisier@math.tamu.edu }\end{array}$ & Tristan Rivière & $\begin{array}{l}\text { ETH, Switzerland } \\
\text { riviere@math.ethz.ch }\end{array}$ \\
\hline Igor Rodnianski & $\begin{array}{l}\text { Princeton University, USA } \\
\text { irod@ math.princeton.edu }\end{array}$ & Wilhelm Schlag & $\begin{array}{l}\text { University of Chicago, USA } \\
\text { schlag@ math.uchicago.edu }\end{array}$ \\
\hline Sylvia Serfaty & $\begin{array}{l}\text { New York University, USA } \\
\text { serfaty@ cims.nyu.edu }\end{array}$ & Yum-Tong Siu & $\begin{array}{l}\text { Harvard University, USA } \\
\text { siu@math.harvard.edu }\end{array}$ \\
\hline Terence Tao & $\begin{array}{l}\text { University of California, Los Angeles, USA } \\
\text { tao@math.ucla.edu }\end{array}$ & Michael E. Taylor & $\begin{array}{l}\text { Univ. of North Carolina, Chapel Hill, USA } \\
\text { met@ math.unc.edu }\end{array}$ \\
\hline Gunther Uhlmann & $\begin{array}{l}\text { University of Washington, USA } \\
\text { gunther@math.washington.edu }\end{array}$ & András Vasy & $\begin{array}{l}\text { Stanford University, USA } \\
\text { andras@math.stanford.edu }\end{array}$ \\
\hline an Virgil Voiculescu & $\begin{array}{l}\text { University of California, Berkeley, USA } \\
\text { dvv@ math.berkeley.edu }\end{array}$ & Steven Zelditch & $\begin{array}{l}\text { Johns Hopkins University, USA } \\
\text { szelditch@math.jhu.edu }\end{array}$ \\
\hline
\end{tabular}

\section{PRODUCTION}

apde@mathscipub.org

Paulo Ney de Souza, Production Manager Sheila Newbery, Production Edito

Silvio Levy, Senior Production Editor

See inside back cover or pjm.math.berkeley.edu/apde for submission instructions.

The subscription price for 2010 is US \$120/year for the electronic version, and \$180/year for print and electronic. Subscriptions, requests for back issues from the last three years and changes of subscribers address should be sent to Mathematical Sciences Publishers, Department of Mathematics, University of California, Berkeley, CA 94720-3840, USA.

Analysis \& PDE, at Mathematical Sciences Publishers, Department of Mathematics, University of California, Berkeley, CA 94720-3840 is published continuously online. Periodical rate postage paid at Berkeley, CA 94704, and additional mailing offices.

APDE peer-review and production is managed by EditFLOW ${ }^{\mathrm{TM}}$ from Mathematical Sciences Publishers.

PUBLISHED BY

mathematical sciences publishers

http://www.mathscipub.org

A NON-PROFIT CORPORATION

Typeset in IATEX $_{\mathrm{E}}$

Copyright @2010 by Mathematical Sciences Publishers 


\section{ANALYSIS \& PDE}

\section{Volume $3 \quad$ No. $2 \quad 2010$}

Polynomials with no zeros on the bidisk

\section{GREG KNESE}

Local wellposedness for the 2+1-dimensional monopole equation Magdalena CZubaK

Regularity of almost periodic modulo scaling solutions for mass-critical NLS and applications DONG LI and XIAOYI ZHANG

Estimées des noyaux de Green et de la chaleur sur les espaces symétriques GILLES CARRON

Lower bounds for resonances of infinite-area Riemann surfaces 